\title{
Bayesian Uncertainty Analysis of Finite Deformation Viscoelasticity
}

\author{
Paul Miles ${ }^{\mathrm{a}}$, Michael Hays ${ }^{\mathrm{a}}$, Ralph Smith ${ }^{\mathrm{b}}$, William Oates $^{\mathrm{a}, *}$ \\ ${ }^{a}$ Florida Center for Advanced Aero Propulsion (FCAAP) \\ Florida $A \& B$ and Florida State University, Department of Mechanical Engineering \\ Tallahassee, FL 32310 \\ ${ }^{b}$ North Carolina State University \\ Department of Mathematics \\ Raleigh, NC 27695
}

\begin{abstract}
The viscoelasticity of the dielectric elastomer, VHB 4910, is experimentally characterized, modeled, and analyzed using Bayesian uncertainty analysis. Whereas these materials are known for their large-field induced deformation and broad applications in smart structures, the rate-dependent viscoelastic effects are not well understood. To address this issue, we quantify both the hyperelastic and viscoelastic constitutive behavior and use Bayesian uncertainty analysis to assess several key modeling attributes. Specifically, we compare an Ogdenbased phenomenological model to a nonaffine hyperelastic model and couple hyperelasticity to both linear and nonlinear viscoelasticity. The utilization of Bayesian statistics is shown to provide insight into quantifying nonlinear viscoelasticity behavior as a function of internal state variables. The results are validated experimentally in the finite deformation regime over a range of stretch rates spanning four orders of magnitude $\left(6.7 \times 10^{-5} \mathrm{~Hz}\right.$ to $\left.0.67 \mathrm{~Hz}\right)$. A unique set of hyperelastic parameters are identified, independent of the stretch rate. In addition, comparisons of the linear and nonlinear viscoelastic models demonstrate a reduction in modeling error by approximately a factor of three. Finally, the viscoelastic time constant is shown to produce an inverse stretch rate power
\end{abstract}

\footnotetext{
*Corresponding Author. Telephone: (850) 645-0139

Email addresses: prm13b@my.fsu.edu (Paul Miles), michael.hays@cummins.com (Michael Hays), rsmith@ncsu.edu (Ralph Smith), woates@fsu.edu (William Oates)
}

Preprint submitted to Mechanics of Materials

July 1, 2015

(C) 2015. This manuscript version is made available under the Elsevier user license http://www.elsevier.com/open-access/userlicense/1.0/ 
law dependence regardless of which hyperelastic model is used.

Keywords: Viscoelasticity, Uncertainty, Dielectric Elastomers, Bayesian

statistics

\section{Introduction}

The utilization of active polymers in adaptive structures is known to provide unique capabilities for real time control of a structure's shape, stiffness, or damping [1]. Knowledge of the viscoelastic constitutive behavior over a broad

5 range of deformation rates becomes particularly important in many applications where dynamic tunability or actuator control is of critical importance. Active polymers such as dielectric elastomers, liquid crystal networks, ionic polymers, and nanocomposites often exhibit complicated viscoelastic characteristics that vary over many orders of magnitude of the stretch rate [2, 3]. The introduction of such a broad range of time scales into a continuum model presents a significant challenge in connecting the underlying material physics with a set of model parameters. Further, in finite deformation regimes, the application of linear or nonlinear viscoelasticity becomes important. The quantification and analysis of model parameter uncertainty is shown to provide important insight towards characterizing this complex material behavior.

Whereas viscoelasticity has been studied extensively [3, 44, 5, 6, 6, ㅁ, 9], there still remains significant challenges in accurately quantifying and predicting rate-dependent, finite deformation over a broad range of elastomer deformation rates. The classic formulation utilizes a combination of Maxwell and

20 Kelvin Voigt spring-dashpot models conceptualized from a more general nonconservative thermodynamic framework [7, 10, 11, 12]. Each discrete linear dashpot is used to quantify rate-dependent stresses as being linear with respect to the stretch rate. Generalizations of this model to three-dimensional thermomechanical deformation with internal order parameters are well summmarized 25 by Gurtin [11] and specific functional forms of the constitutive model have been implemented in finite element codes by Holzapfel and Gasser where they as- 
sumed linear viscoelasticity coupled to finite deformation [13].

Here we adopt the nonlinear mechanics and thermodynamics approach summarized in 10, 12] and analyze model and experimental uncertainty when quantifying a set of hyperelastic and viscoelastic parameters. The form of the dissipative energy function and the entropy generation function used in quantifying viscoelasticity are analyzed through comparisons with rate dependent stressstretch measurements and Bayesian uncertainty analysis. Questions often arise in finite deformation mechanics as to the appropriate model for characterizing the viscous nature of the polymer network during large deformation. It is shown that if the dissipative function is assumed to be proportional to the hyperelastic function (e.g., a form of nonlinear viscoelasticity), the model accuracy improves by a factor of three relative to a linear viscoelastic model for our particular elastomer (VHB 4910 made by 3M).

Classical hyperelasticity is motivated by statistical mechanics which describes a polymer network in terms of changes in configurational entropy [14]. In the undeformed configuration, the polymer is typically assumed to be a Gaussian network (completely random) which results in a neo-Hookean configurational entropy model. Upon large deformation, the polymer chains align reducing the 45 configurational entropy beyond configurations that can be assumed Gaussian. Such behavior is often described by a Langevin function [14]. In most cases, this function only qualitatively matches data. Alternatively, polynomial approximations in terms of principal stretches are used to achieve quantitative predictions of data. Such models include the hyperelastic Ogden, Mooney-Rivlin, or Arruda-Boyce models [15]. Critical to the development of these constitutive relations is the assumption of affine deformation in which all cross-linked points in the polymer network displace proportional to the macroscopic stretch. It can be argued that the entanglement and crosslinked network structure of many polymers contain network segments with varying pre-tension that do not deform in 55 an affine manner.

Recent research has focused on using nonaffine deformation to describe reversible hyperelastic material behavior [2, 16, 17]. In particular, Davidson and 
Goulbourne showed that decomposing hyperelastic stresses into a crosslinked stress and an entanglement stress leads to an accurate prediction of elastomer deformation for both tensile and compressive deformation in different elastomer materials [16]. Here we first compare this approach to a six parameter Ogden model using Bayesian statistics. Second, we couple hyperelasticity with finite deformation linear and nonlinear viscoelastic models. Bayesian uncertainty analysis is shown to be insightful in assessing assumptions that go into the constitutive model and the choice of linear versus nonlinear viscoelastic models. The robustness of the models is illustrated through quantifying probability distributions of each parameter and how these distributions propagate through the model leading to credible and prediction intervals of stress predictions for a given stretch and stretch rate.

The differences in these models are considered in Section 5 where we explore the coupling between hyperelasticity and viscoelasticity to better understand important rate-dependent material parameters governing hysteresis in elastomers. Specifically, we compare the impact of assumptions associated with affine versus nonaffine polymer chain deformation and extensions to linear and nonlinear viscoelasticity. It is shown that a nonaffine hyperelastic model can give practically the same prediction of finite deformation over a broad range of stretch rates (four orders of magnitude) using half the number of parameters in comparison to a six parameter Ogden hyperelastic model. Further improvements in accuracy are achieved using nonlinear viscoelasticity which assumes dissipation is proportional to the hyperelastic energy function instead of a proportionality to a neo-Hookean free energy density function (linear viscoelasticity).

The numerical algorithm used to sample the set of material parameters, in conjunction with Bayesian statistics, is a Markov Chain Monte Carlo (MCMC) algorithm that uses the Delayed Rejection Adaptive Metropolis (DRAM) method distribution for parameters based on a sum-of-squares error between the model and data. The criteria for proposing and accepting a parameter value is based on a distribution rather than simply a decrease in error. From the accepted 
parameter values, Bayesian statistics allow us to quantify the model parameter distributions instead of quantifying fixed values. Those distributions can subsequently be used to calculate uncertainty propagation in the prediction of stress for a given stretch and stretch rate. Whereas this method has been used in a broad range of disciplines including uncertainty quantification of atomistic potentials [20], computational fluid dynamics [21], weather prediction [22], and engineering structures and design [23, 24]; less work has been focused on uncertainty analysis of nonlinear continuum based constitutive models [25, 26].

In Section 2, we describe the experimental methods and summarize the experimental data. In Section 3, the viscoelastic model is presented including specific hyperelastic and energy dissipation functions used to predict rate dependent, finite deformation material behavior. Bayesian statistics are introduced in Section 4 to assess model parameter uncertainty using the different models. Concluding remarks are given in Section 6.

\section{Experimental Methods}

The testing was done using an MTS Insight $1 \mathrm{kN}$ load frame and a $5 \mathrm{~N}$ load cell. Test specimens were made from rolls of VHB 4910 with a nominal thickness of $1 \mathrm{~mm}$. Each specimen was cut by hand, maintaining approximately uniform width along the entire length of the sample. The initial length of each specimen was defined by the grip separation distance between the load frame and load cell, which was nominally $30 \mathrm{~mm}$. The specimen was secured in the MTS machine, in a slightly relaxed state to avoid any viscoelastic creep prior to testing. The uncertainty in the true gauge length was accounted for in the data processing by defining the true gauge length at the point of initial non-zero tensile stress.

Each specimen was put through several load/unload cycles where it was stretched to six times its initial length $(\lambda=6)$ and then unloaded back to the initial state; all at a constant stretch rate. This process was repeated 12 times in immediate succession as illustrated in Figure 1(a-c). Experiments were performed at multiple stretch rates, from $6.7 \times 10^{-5} \mathrm{~Hz}$ to $0.67 \mathrm{~Hz}$. The stretch 
rate was defined as the speed at which the specimen was moving divided by the initial length $\left(\frac{d \lambda}{d t}=\frac{\dot{x}}{L_{0}}\right)$. In our test cases, five test specimens would complete all 12 cycles at a single stretch rate. We performed six test cases at different stretch rates which resulted in 26 different data sets $(5$ samples each at $0.0472 \mathrm{~Hz}, 0.10 \mathrm{~Hz}, 0.335 \mathrm{~Hz}, 0.50 \mathrm{~Hz}$, and $0.67 \mathrm{~Hz} ; 1$ sample at $6.7 \times 10^{-5} \mathrm{~Hz}$ ).

\subsection{Experimental Results}

The cyclic fatigue behavior over a range of stretch rates is illustrated in Figure 1 A significant reduction in the stress as the number of cycles increases can be seen in Figure $1(\mathrm{a}-\mathrm{c})$. This reduction in peak stress per cycle diminishes at about 8 cycles and the stress to stretch relationship approaches steady state hysteresis. The modeling of the data was performed on the $12^{\text {th }}$ cycle at which
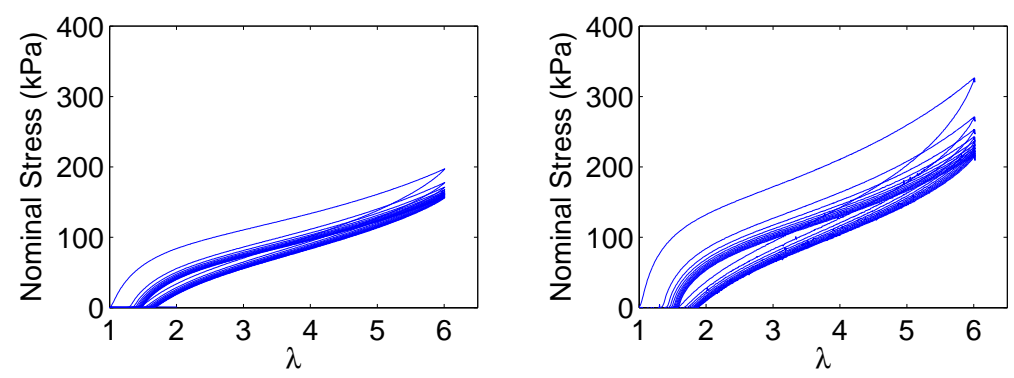

(a) $\frac{d \lambda}{d t}=0.0472 \mathrm{~Hz}$

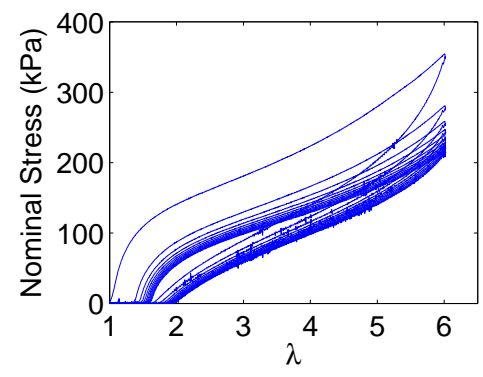

(b) $\frac{d \lambda}{d t}=0.335 \mathrm{~Hz}$

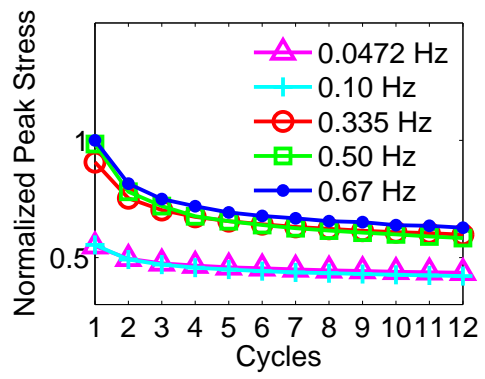

(c) $\frac{d \lambda}{d t}=0.67 \mathrm{~Hz}$

(d) Peak stress decay

Figure 1: (a-c) Cyclic loading over 12 cycles and (d) decay of peak stress per cycle. The decay of the peak stress is normalized by the maximum stress when $\dot{\lambda}=0.67 \mathrm{~Hz}$. Similar results are not shown for tests performed at $0.10 \mathrm{~Hz}$ and $0.50 \mathrm{~Hz}$. 

and hysteresis are observed as the stretch rate increases; however, accurate model predictions of this behavior are shown to be non-trivial as described through model analysis in Section 3 .

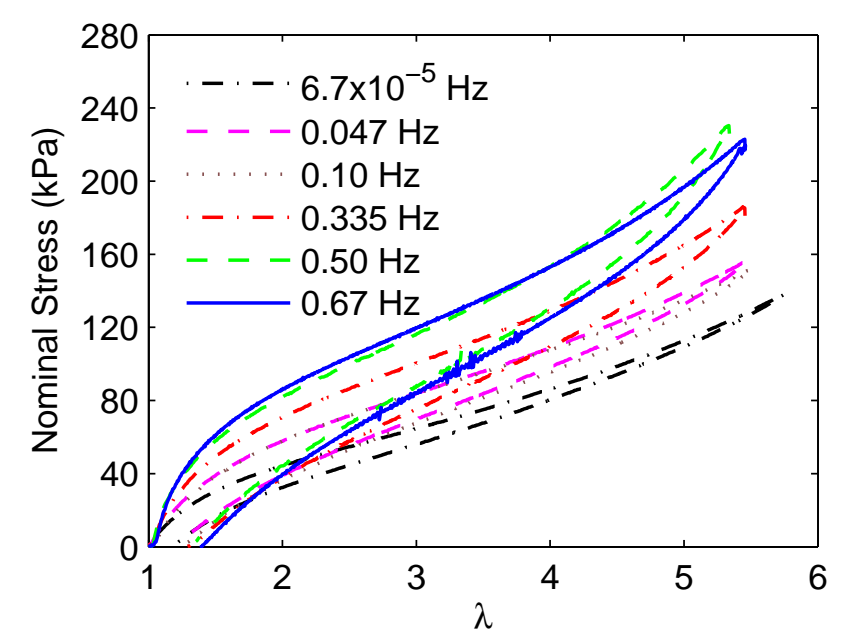

Figure 2: Comparison of the viscoelastic behavior for the $12^{\text {th }}$ cycle for each stretch rate (Note: $\frac{d \lambda}{d t}=6.7 \times 10^{-5} \mathrm{~Hz}$ was only run for one cycle, and the stretch $\lambda=1$ has been adjusted by subtracting out viscoplastic strain from cyclic loading.)

\section{Mathematical Models}

We summarize the hyperelastic and viscoelastic governing equations in this section. Internal variables associated with rate-dependent dissipation are developed following the general thermodynamic framework outlined in [10, 11, 12]. The origins of the dissipative equation governing internal loss is based on an unknown function of entropy generation derived from the second law of thermo- 
dynamics. This relationship is highlighted to provide guidance to the integration of the hyperelastic energy functions and coupling with viscoelastic dissipative energy functions. Through this approach, we develop linear and nonlinear forms of the viscoelastic stress and analyze its uncertainty using Baysian statistics in Section 4

\subsection{Finite Deformation Energy Relations}

The following thermodynamic framework starts with the inclusion of thermal effects, but will later focus on model validation under the assumption of isothermal deformation. The total energy density function including dissipation 155 is given by

$$
\psi=\psi_{\infty}\left(F_{i K}, \Theta\right)+\Upsilon\left(F_{i K}, \Theta, \Gamma_{i K}^{\alpha}\right)
$$

per reference volume where $\psi_{\infty}$ is the conserved, hyperelastic free energy function and $\Upsilon$ is a dissipative energy function. The hyperelastic free energy is a function of the deformation gradient $F_{i K}$ and temperature $\Theta$ while $\Upsilon$ includes $\Gamma_{i K}^{\alpha}$, which are a set of internal variables $(\alpha=1, \ldots, n$ non-measurable internal states) that contribute to dissipation during rate dependent deformation.

Since elastomers typically undergo incompressible deformation, a penalty term is added to the free energy, so that the total free energy density is

$$
\hat{\psi}=\psi-p(J-1)
$$

where $p$ is the unknown Lagrange multiplier, which represents a hydrostatic stress and $J=\operatorname{det}\left(F_{i K}\right)$. Incompressible deformation is thus described by $J=1$.

It will be shown that the work conjugate variable to the deformation gradient is the nominal stress

$$
s_{i K}=\frac{\partial \hat{\psi}}{\partial F_{i K}}=\frac{\partial \psi_{\infty}}{\partial F_{i K}}-p J H_{i K}+\frac{\partial \Upsilon}{\partial F_{i K}},
$$

where we have used the identity $\frac{\partial J}{\partial F_{i K}}=J H_{i K}$ and $H_{i K} F_{j K}=\delta_{i j}[15$. 
The work conjugate variable for $\Gamma_{i K}^{\alpha}$ is

$$
Q_{i K}^{\alpha}=-\frac{\partial \hat{\psi}}{\partial \Gamma_{i K}^{\alpha}}=-\frac{\partial \Upsilon}{\partial \Gamma_{i K}^{\alpha}}
$$

170

entropy per mass and $\Theta$ is temperature, we obtain

$$
\rho^{0} \dot{\hat{\psi}}=s_{i K} \dot{F}_{i K}+\rho^{0} r-Q_{I, I}-\rho^{0} \Theta \dot{S}-\rho^{0} S \dot{\Theta} .
$$

This form of the first law is combined with the second law, given here in the Lagrangian frame as

$$
\rho^{0} \dot{S} \geq \frac{\rho^{0} r}{\Theta}-\frac{1}{\rho^{0}}\left(\frac{Q_{I}}{\Theta}\right)_{, I} .
$$

Prior to combining (6) and (77), we take the time derivative of the total energy 185 function $\hat{\psi}$,

$$
\dot{\hat{\psi}}=\frac{\partial \hat{\psi}}{\partial F_{i K}} \dot{F}_{i K}+\frac{\partial \hat{\psi}}{\partial \Theta} \dot{\Theta}+\frac{\partial \hat{\psi}}{\partial \Gamma_{i K}^{\alpha}} \dot{\Gamma}_{i K}^{\alpha},
$$

based upon the state variables given in (11). A substitution of this relation into the first and second law equations (6) and (7) yields

$$
\left(s_{i K}-\frac{\partial \hat{\psi}}{\partial F_{i K}}\right) \dot{F}_{i K}-\rho^{0}\left(S+\frac{\partial \hat{\psi}}{\partial \Theta}\right) \dot{\Theta}-\frac{\partial \hat{\psi}}{\partial \Gamma_{i K}^{\alpha}} \dot{\Gamma}_{i K}^{\alpha}-\frac{Q_{I} \Theta_{, I}}{\Theta^{2}} \geq 0,
$$


which confirms the work conjugate relation in (3). The third term gives the viscoelastic stress from (44). The additional work conjugate relation on entropy ${ }_{190}$ is $S=-\frac{\partial \hat{\psi}}{\partial \Theta}$. The second law then requires that

$$
-\frac{\partial \hat{\psi}}{\partial \Gamma_{i K}^{\alpha}} \dot{\Gamma}_{i K}^{\alpha}-\frac{Q_{I} \Theta_{, I}}{\Theta^{2}} \geq 0 .
$$

These two terms describe the entropy production. The second relation is normally restricted to be positive definite by allowing the heat flux to be $Q_{I}=$ $-\kappa_{I J} \Theta_{, J}$ where the thermal conductivity tensor $\left(\kappa_{I J}\right)$ is positive definite. In cases where the thermal gradients are negligible but viscoelastic effects are present, the first term on the right hand side must be positive definite.

We assume that thermal gradients are negligible and the viscoelasticity is the only source of entropy production. Following Peng et al. [12], we assume entropy production is a function of the time rate of change of the internal state variable and the deformation gradient,

$$
-\frac{\partial \hat{\psi}}{\partial \Gamma_{i K}^{\alpha}} \dot{\Gamma}_{i K}^{\alpha}=Q_{i K}^{\alpha} \dot{\Gamma}_{i K}^{\alpha}=F\left(\dot{\Gamma}_{i K}^{\alpha}, F_{i K}\right) \geq 0,
$$

200 $F\left(\dot{\Gamma}_{i K}^{\alpha}, F_{i K}\right)$ are unknown. We assume the entropy production can be approximated by a Taylor expansion of the form

$$
F\left(\dot{\Gamma}_{i K}^{\alpha}, F_{i K}\right)=\eta^{\alpha} \dot{\Gamma}_{i K}^{\alpha} \dot{\Gamma}_{j L}^{\alpha}+\cdots,
$$

where $\eta^{\alpha}$ must be positive definite to satisfy the second law. By assuming relative small rates of change of the internal state, we neglect any higher order terms in (12) and substitute this relation into (11) to obtain the equation

$$
\eta^{\alpha} \dot{\Gamma}_{i K}^{\alpha} \dot{\Gamma}_{i K}^{\alpha}-Q_{i K} \dot{\Gamma}_{i K}^{\alpha}=0
$$

which leads to $Q_{i K}^{\alpha}=\eta^{\alpha} \dot{\Gamma}_{i K}^{\alpha}$. This is a generalized viscoelastic constitutive law analogous to a spring-dashpot model in one dimension [15]; however, it is important to note that this does not necessarily mean that the viscoelastic behavior is linear. It only states that the rate of change of the internal state is 

a dissipation function that is analogous to the neo-Hookean hyperelastic energy function. It will be shown that if the stretch is significant such that the neo-Hookean model breaks down, the viscoelastic behavior is less accurately modeled using the neo-Hookean viscous proportionality. 
Implementation of the linear viscoelastic model for comparison to the nonlinear model requires modifying (15) by substitution of $\Upsilon_{L}$ instead of $\Upsilon_{N L}$ to solve for the internal state using

$$
\eta^{\alpha} \dot{\Gamma}_{i K}^{\alpha}+\gamma^{\alpha} \Gamma_{i K}^{\alpha}=\gamma^{\alpha} F_{i K}
$$

It is often preferable to re-write this equation in terms of the viscoelastic stress given by (4). A substitution of this stress into (15) and taking the time derivative of the entire equation leads to

$$
\dot{Q}_{i K}^{\alpha}+\frac{1}{\tau^{\alpha}} Q_{i K}^{\alpha}=\gamma^{\alpha} \dot{F}_{i K}
$$

where $\tau^{\alpha}=\frac{\eta^{\alpha}}{\gamma^{\alpha}}$. This linear viscoelastic stress equation will be coupled with the calculation of the hyperelastic stress in (3) which results in the total stress

$$
s_{i K}=\frac{\partial \psi_{\infty}}{\partial F_{i K}}-p J H_{i K}+\sum_{\alpha} Q_{i K}^{\alpha}
$$

since in the linear viscoelastic model we have $Q_{i K}^{\alpha}=\frac{\partial \Upsilon}{\partial F_{i K}}=-\frac{\partial \Upsilon}{\partial \Gamma_{i K}^{\alpha}}$.

In summary, the nonlinear viscoelastic model requires solving (3) and (15) where $\Upsilon \rightarrow \Upsilon_{N L}$. In the linear viscoelastic model, the total stress requires solving (19) together with (18). Solution of these equations requires specifying a hyperelastic energy function. In the following subsection, we introduce the Ogden and nonaffine hyperelasticity functions $\left(\psi_{\infty}\right)$ to complete the set of relations required to quantify rate-dependent stresses.

\subsection{Hyperelastic Energy Functions}

Two hyperelastic energy functions are introduced for integration into the viscoelastic model and its coupling with the dissipative energy functions given by (14) and (16). First, the Ogden hyperelastic model is considered. It is written in terms of the principal stretches $\lambda_{i}$ for the principal directions $i=1$ to 3 . This hyperelastic energy is

$$
\psi_{\infty}^{O}=\sum_{d=1}^{3} \frac{\mu_{d}}{\alpha_{d}}\left(\lambda_{1}^{\alpha_{d}}+\lambda_{2}^{\alpha_{d}}+\lambda_{3}^{\alpha_{d}}-3\right)
$$


where $\mu_{d}$ are shear moduli with the effective shear modulus, $\mu=\sum_{d=1}^{3} \mu_{d}$, and $\alpha_{d}$ are unitless constants [15]. The model is physically constrained so that $\sum_{d=1}^{3} \mu_{d} \alpha_{d} \geq 0$.

In comparison, the nonaffine model combines the effect of a crosslinked network with entanglement effects described by the free energy [16]

$$
\psi_{\infty}^{N}=\frac{1}{6} G_{c} I_{1}-G_{c} \lambda_{\max }^{2} \ln \left(3 \lambda_{\max }^{2}-I_{1}\right)+G_{e} \sum_{j}\left(\lambda_{j}+\frac{1}{\lambda_{j}}\right)
$$

where $G_{c}$ is the crosslink network modulus, $G_{e}$ is the plateau modulus, $\lambda_{\max }$ is the maximum stretch of the effective affine tube, and $I_{1}=\lambda_{i} \lambda_{i}$ is the first stretch invariant where summation on $i$ is implied.

These two energy functions are used to determine the hyperelastic stress originally defined in (3). Numerical analysis includes comparison of data with both hyperelastic energy functions coupled with the linear viscoelastic dissipation function (16) and also the nonaffine hyperelastic energy function coupled with the nonlinear viscoelastic dissipation function (14).

\subsection{Summary of Models and Parameters}

We summarize here the models and parameters whose properties, sensitivities, and uncertainties we will investigate using Bayesian model calibration techniques. We denote calibration parameters by $\theta$ to differentiate them from parameters whose values are assumed fixed and known.

\section{Linear Ogden Model}

The linear viscoelastic Ogden model is given by (16) and (20) and has eight calibration parameters

$$
\theta=\left[\mu_{d}, \alpha_{d}, \tau, \gamma\right], d=1,2,3,
$$

which are identified individually at each strain rate. As indicated in Section 3.2 the hyperelastic parameters $\mu_{p}$ and $\alpha_{p}$ must satisfy the physical constraint

$$
\sum_{d=1}^{3} \mu_{d} \alpha_{d} \geq 0 .
$$


We also consider the performance of the Ogden model when the six hyperelastic parameters $\mu_{d}, \alpha_{d}$ are estimated at the slowest stretch rate and fixed for subsequent experiments. In this case, the two viscoelastic parameters

$$
\theta=[\tau, \gamma]
$$

are calibrated using Bayesian analysis.

Linear Nonaffine Model

The linear viscoelastic nonaffine model is given by (16) and (21) and has the

285

Nonlinear Nonaffine Model

In this case, the viscoelastic model has six parameters

$$
\theta=\left[G_{c}, G_{e}, \lambda_{\max }, \eta, \beta, \gamma\right]
$$

from (14) and (21). The viscoelastic model obtained with fixed hyperelasltic parameters has the three calibration parameters

$$
\theta=[\eta, \beta, \gamma] .
$$

Similar results were obtained when combining linear viscoleasticity with the Ogden and nonaffine hyperelastic models (see Section 5). Therefore, analysis of the nonlinear viscoelasticity was only performed in combination with the nonaffine model. 


\section{Bayesian Statistical Analysis}

Bayesian model calibration is based on the tenet that calibration parameters

typically exhibit uncertainty due to model discrepancies and observation errors associated with data used to estimate parameters. To quantify this uncertainty, parameters are taken to be random variables having associated densities or distributions. Details regarding this approach are provided in Chapter 8 of [19].

We employ the statistical model

$$
s_{33}^{\text {data }}(i)=s_{33}(i ; \theta)+\varepsilon_{i}, i=1, \cdots, N,
$$

where $s_{33}^{\text {data }}(i)$ and $\varepsilon_{i}$ are random variables respectively denoting the $i^{\text {th }}$ experimental data point and associated observation error. Here $s_{33}(i ; \theta)$ is the parameter-dependent model response given by (3) and $\theta$ is given by (22) -(28) for the considered models. We note that the observation errors include measurement errors inherent to the MTS machine as well as variability between tests run at differing stretch rates. We also point out that a different specimen was used for each stretch rate due to the irreversible material evolution illustrated in Figure 1. Lastly, it may also include model discrepancies due to limitations in physical models.

\subsection{Bayesian Model Calibration}

315 Bayesian model calibration is based on the use of Bayes' relation

$$
\pi\left(\theta \mid s_{33}^{\text {data }}\right)=\frac{p\left(s_{33} \mid \theta\right) \pi_{0}(\theta)}{\int_{\mathbb{R}^{p}} p\left(s_{33} \mid \theta\right) \pi_{0}(\theta) d \theta}
$$

to determine the posterior density $\pi\left(\theta \mid s_{33}^{\text {data }}\right)$, which quantifies the probability of observing parameter values $\theta$ given the data $s_{33}^{\text {data }}$. Here $\pi_{0}(\theta)$ denotes the prior density, which quantifies a priori knowledge about the parameters before data is collected. This prior is updated using the likelihood $p\left(s_{33} \mid \theta\right)$, which 320 incorporates information provided by the model and data. The denominator normalizes the density to have an area of unity.

Because we assume no prior knowledge about parameters, other than their support - e.g., positivity for $\tau$ and $\gamma$,- we employ noninformative priors having 
a value of one on the feasible parameter space. This ensures that prior distributions do not adversely inform the posterior through incorrect initial information.

To construct a likelihood function, we must make assumptions regarding the observation errors $\varepsilon_{i}$. With the assumption that observation errors are independent and identically distributed (iid) and $\varepsilon_{i} \sim N\left(0, \sigma^{2}\right)$, the likelihood is

$$
p\left(s_{33} \mid \theta\right)=e^{-\sum_{i=1}^{N}\left[s_{33}^{\text {data }}(i)-s_{33}(i ; \theta)\right]^{2} / 2 \sigma^{2}} .
$$
ever, this is not typically known a priori, thus necessitating the use of adaptive 
quadrature techniques. Tensored quadrature rules are not effective for the $p=8$ case so adaptive sparse grid techniques would be required.

We avoid the issues associated with adaptive quadrature by employing sampling-based Metropolis algorithms whose stationary distribution is the posterior density $\pi\left(\theta \mid s_{33}^{\text {data }}\right)$. Specifically, we employ the Delayed Rejection Adaptive Metropolis (DRAM) algorithm developed in [18, 27] and detailed in [19] with code provided at the website [28]. When implementing the linear Ogden model, we discard proposed parameter sets that violate the constraint (23). The convergence of the algorithm with discarded chain elements can be motivated as follows. The convergence of the non-Markovian DRAM algorithm is essentially established using a decaying adaptation condition. That condition is maintained when discarding chain elements resulting from parameter values that violate (23). Finally, we employ the DRAM algorithm, rather than a standard Gibbs sampler, to accommodate the highly correlated parameter sets.

\subsection{Credible and Prediction Intervals}

To quantify model uncertainty, due to parameter uncertainty and observation errors, one can construct credible and prediction intervals for responses or statistical quantities of interest. Credible intervals can be constructed by propagating a statistically significant number of posterior parameter values, given by (30), through the model to provide response distributions.

To construct prediction intervals, one propagates both posterior parameter values and observation errors $\varepsilon_{i} \sim N\left(0, \sigma^{2}\right)$ through the models. The variance, $375 \sigma^{2}$, is also inferred through Bayesian model calibration techniques. Because prediction intervals include both parameter and observation uncertainty, they quantify the probability of observing future numerical predictions or experimental observations. Hence they quantify the model's predictive capability. Discussion regarding the construction and interpretation of Bayesian credible and prediction intervals are provided in Section 9.4 of [19]. Details regarding conditions under which prediction intervals can be employed for extrapolation are provided in [29]. In particular, we assume that future experiments are performed under 
the same conditions used for inference and that only the independent variables

- here the stretch rates - are changed.

385

390 model, the six parameters (27) in the nonlinear nonaffine model, and the five parameters (25) in the linear nonaffine model. The objective is twofold: (i) construct marginal densities, which quantify inherent parameter uncertainties and (ii) construct pairwise plots that elucidate linear or nonlinear correlation be410

\section{Model Analysis and Results}

The model analysis is guided by the following objectives.

(i) Quantify the relative accuracy by comparing errors produced by the linear Ogden, nonlinear nonaffine, and linear nonaffine models with the combined viscoelastic and hyperelastic parameter sets (22), (25) and (27). Use Bayesian analysis to quantify uncertainties associated with parameters and indicate the degree to which parameters can be uniquely determined from the data - see Section 5.1 .

(ii) Determine the degree to which calibration of the three models can be simplified, and predictive capabilities maintained over the range of stretch rates, by fixing hyperelastic parameters at values determined for the slowest stretch rate. This is motivated by the Bayesian analysis of Section 4.1 and is based on the property that hyperelastic stress is defined to be a function only of the deformation and temperature - see (10) - whereas viscoelastic stress accounts for the observed hysteresis and is greatly in-

(iii) Quantify the degree to which the time constant $\tau$ exhibits a functional dependence on $\frac{d \lambda}{d t}-$ see Section 5.3 .

\subsection{Model Analysis with Viscoelastic and Hyperelastic Calibration Parameters}

We first employ the Bayesian model calibration framework, outlined in Section 4.1. to construct densities for the eight parameters (22) in the linear Ogden tween parameters, which can indicate those parameters that are nonidentifiable in the sense that they cannot be uniquely determined from the data. 
We illustrate the first two objectives for the six parameter nonlinear nonaffine model. The application of the DRAM algorithm to the lowest frequency stretch rate data, $\frac{d \lambda}{d t}=6.7 \times 10^{-5} \mathrm{~Hz}$, yielded the parameter chains shown in Figure 3. We note that with $5 \times 10^{6}$ evaluations, the chain is burned-in in the sense that it has converged to the posterior density. Qualitatively, this can be established by observing that the chains have the appearance of approximately white noise with no significant jumps in the mean behavior or regions of stagnation. Statistical acceptance and convergence tests employed in DRAM, and more generally Metropolis algorithms, are detailed in [18, 27, 30].

A kernel density estimation (KDE) algorithm is used to construct the marginal densities, shown in Figure 4 from the burned-in parameter chains. It is observed that whereas the marginal density for $\lambda_{\max }$ is approximately Gaussian, the other five densities are highly non-Gaussian. This illustrates the fact that the Metropolis algorithm can be used to construct general densities.

The pairwise plots in Figure 5 quantify the correlation between parameters.

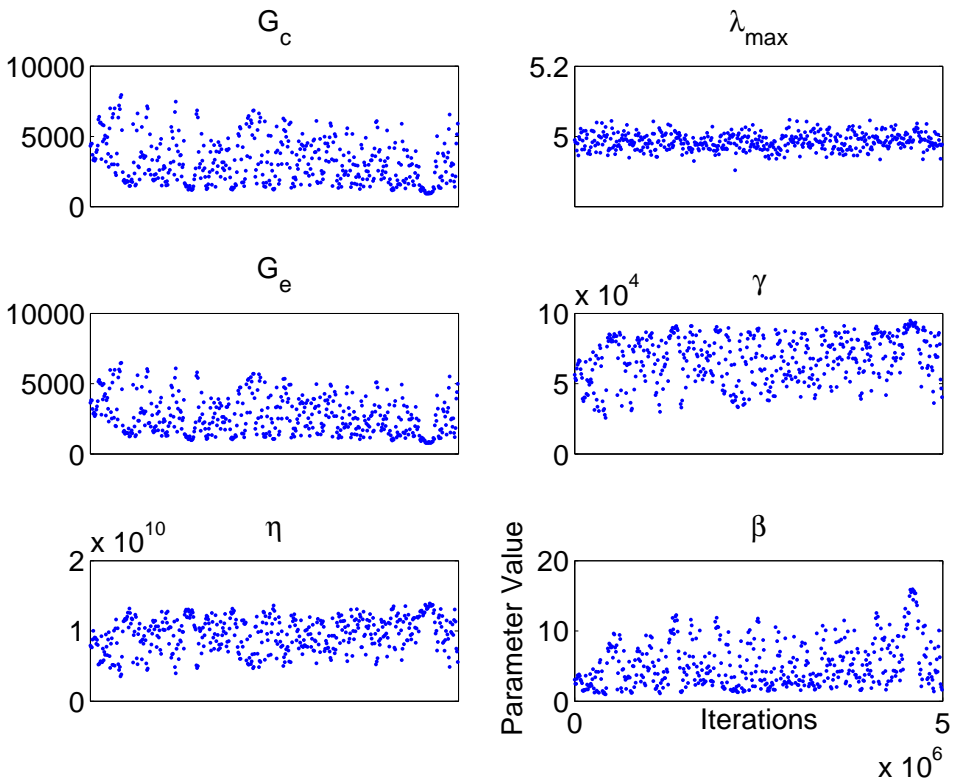

Figure 3: Parameter chains obtained with $5 \times 10^{6}$ realizations of the nonlinear nonaffine model to demonstrate burn-in. 

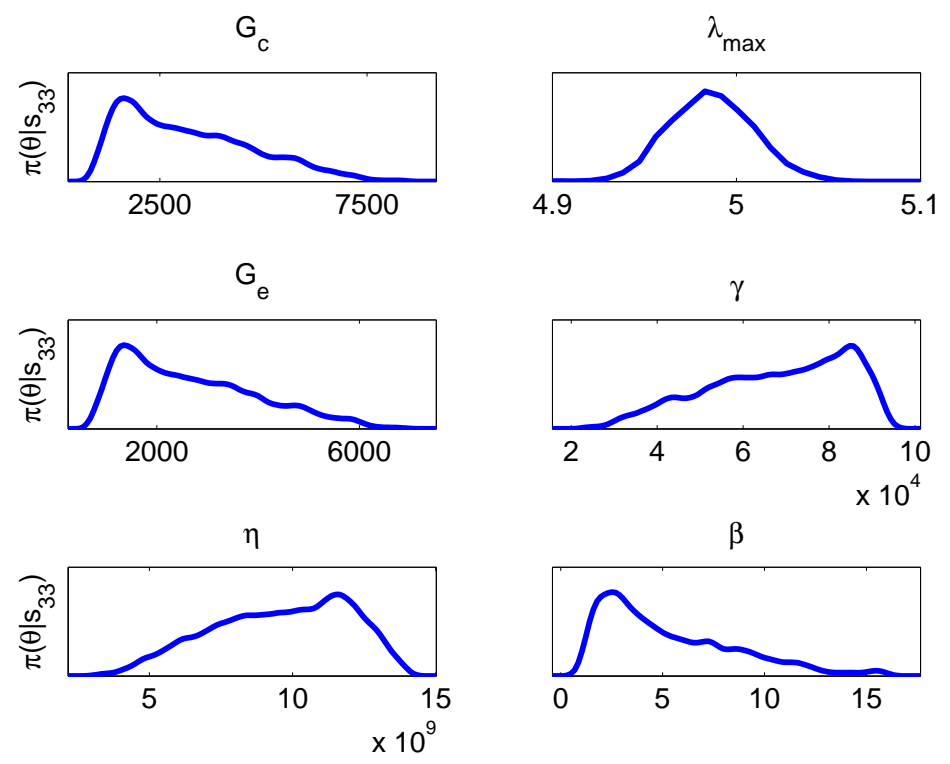

Figure 4: Marginal posterior parameter densities for the nonlinear nonaffine viscoelastic model at the stretch rate $\frac{d \lambda}{d t}=6.7 \times 10^{-5} \mathrm{~Hz}$.

The nearly single-valued linear correlation between the crosslink modulus $G_{c}$ and plateau modulus $G_{e}$ indicate that they are not identifiable in the sense that they can be uniquely determined by the data since a single value for one can be used to define the other. The viscoelastic parameters $(\eta, \gamma)$ exhibit a similar nearly single-valued linear correlation. Furthermore, the pairs $\left(G_{c}, \beta\right)$, $\left(G_{c}, \beta\right)$ and $\left(G_{e}, \gamma\right)$ exhibit nearly single-valued nonlinear correlations. This suggests non-trivial coupling among the entanglement and crosslink free energy density and energy dissipation. Further analysis is required to determine if these correlations can be utilized to reduce models through assessment of the underlying polymer network evolution during rate-dependent deformation. Finally, we note that the maximum stretch, $\lambda_{\max }$, appears to be independent from the other parameters.

Similarly, the pairwise plots for the Ogden model illustrate that the eight 440 parameters (22) are highly correlated, with single values, and hence are not jointly identifiable. 

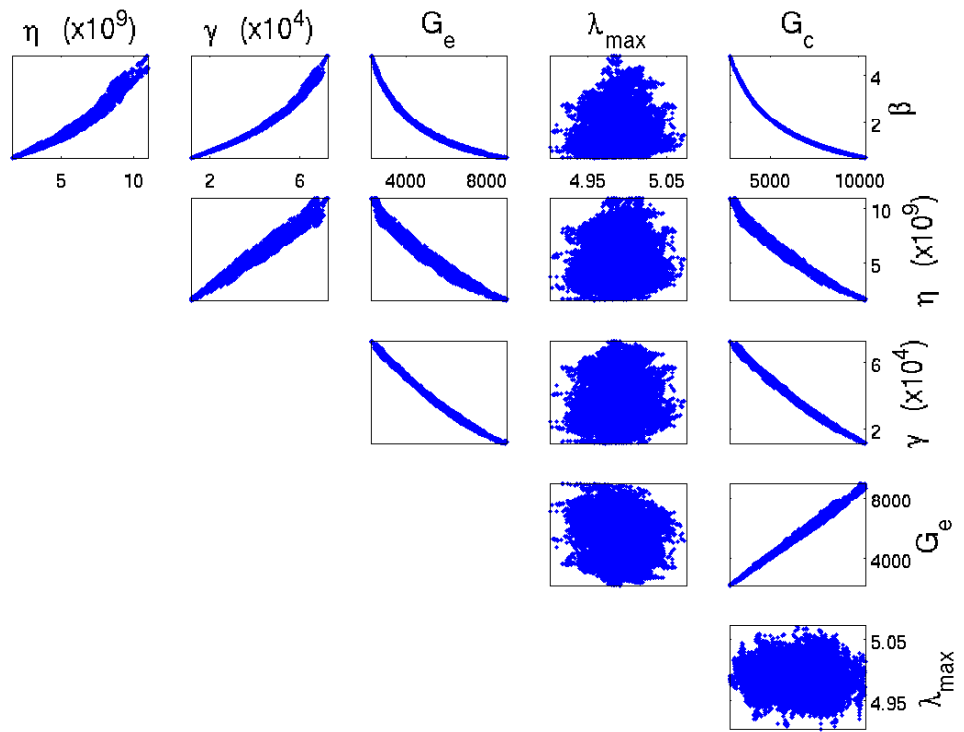

Figure 5: Joint sample points for parameters in the nonlinear nonaffine model for the $\frac{d \lambda}{d t}=$ $6.7 \times 10^{-5} \mathrm{~Hz}$ stretch rate with $\eta$ scaled by $1 \times 10^{9}$ and $\gamma$ scaled by $1 \times 10^{4}$ for formatting purposes.

Whereas Bayesian techniques can be used to construct densities for nonidentifiable parameters, if one employs a tight prior density, optimization or frequentist techniques will generally fail since multiple parameter values yield the same maximum likelihood or minimum ordinary least squares values.

This lack of identifiability is typically addressed in one of two ways. In the first, one uses physical analysis to reformulate or reduce the model in a manner that eliminates non-identifiable parameters. Alternatively, one can fix non-identifiable parameters at physically reasonable nominal values. We employ the latter strategy in Section 5.2 where we fix the hyperelastic parameters $G_{e}, G_{c}, \lambda_{\max }$ at the mean values determined at the slowest stretch rate when calibrating and validating the model at faster stretch rates.

The mean parameter values, computed as sample means from the chains, are compared in Table 1 with mean values computed at the fast stretch rates ${ }_{455} \frac{d \lambda}{d t}=0.0472 \mathrm{~Hz}$ to $\frac{d \lambda}{d t}=0.67 \mathrm{~Hz}$. It is observed that the mean values for the hyperelastic parameters $G_{c}, G_{e}$ and $\lambda_{\max }$ are nearly constant for all stretch 
Table 1: Mean parameter values for the nonlinear nonaffine model estimated at the rates $\frac{d \lambda}{d t}$.

\begin{tabular}{c|c|c|c|c|c|c}
\hline \hline \multicolumn{7}{c}{ Nonlinear Nonaffine Model } \\
\hline \hline$\frac{d \lambda}{d t}$ & $G_{c}(\mathrm{kPa})$ & $\lambda_{\max }$ & $G_{e}(\mathrm{kPa})$ & $\tau(\mathrm{s})$ & $\gamma(\mathrm{kPa})$ & $\beta$ \\
\hline \hline $6.7 \times 10^{-5} \mathrm{~Hz}$ & 5.52 & 4.99 & 4.64 & $1.41 \times 10^{5}$ & 45.5 & 2.11 \\
\hline $0.0472 \mathrm{~Hz}$ & 4.99 & 4.89 & 4.39 & 87.8 & 59.9 & 3.73 \\
\hline $0.10 \mathrm{~Hz}$ & 4.95 & 5.26 & 3.80 & 35.9 & 53.9 & 3.89 \\
\hline $0.335 \mathrm{~Hz}$ & 5.19 & 4.36 & 5.48 & 21.6 & 64.4 & 4.91 \\
\hline $0.50 \mathrm{~Hz}$ & 5.25 & 3.91 & 7.04 & 50.4 & 31.4 & 4.30 \\
\hline $0.67 \mathrm{~Hz}$ & 5.01 & 4.30 & 5.56 & 15.6 & 48.0 & 4.95 \\
\hline \hline
\end{tabular}

rates whereas the means for the viscoelastic parameters vary significantly with $\eta$ changing by three orders of magnitude. This is consistent with the property that the hyperelastic stress is a function only of deformation and temperature whereas the viscoelastic stress accounts for losses and hysteresis, which are highly dependent on stretch rates. When combined with the observation that the hyperelastic and viscoelastic parameters are not jointly identifiable, this further motivates fixing the hyperelastic parameters at the low stretch rate values for subsequent model calibration and validation.

The mean parameter values, computed using analogous Bayesian analysis, for the linear nonaffine and Ogden models are respectively compiled in Tables 2 and 3. Both exhibit more variability among the hyperelastic parameters than observed for the nonlinear nonaffine model with $\mu_{3}$ in the Ogden model varying by two orders of magnitude.

To quantify the accuracy of each model, we normalize the sum-of-square residuals by the number of data points $N$ to obtain the errors $e_{M C M C}=$ $\frac{1}{N} \sum_{i=1}^{N}\left[s_{33}^{\text {data }}(i)-s_{33}(i ; \bar{\theta})\right]^{2}$ reported in Table 4 For each model, the solution $s_{33}(i ; \bar{\theta})$ was computed using the mean parameter values $\bar{\theta}$ obtained using the DRAM algorithm. We noted that these results further illustrate that the nonlinear nonaffine model is the most accurate of the three considered models. 
When combined with the observation that the nonaffine model is considered less phenomenological than the more conventional Ogden model [15, 16], this renders it advantageous for material characterization.

\subsection{Model Analysis with only Viscoelastic Calibration Parameters}

Based on the Bayesian model calibration results reported in Section 5.1 we now fix the hyperelastic parameters at the mean values obtained at the slowest stretch rate $\frac{d \lambda}{d t}=6.7 \times 10^{-5} \mathrm{~Hz}$ and consider the nonlinear and linear nonaffine models and linear Ogden model at higher stretch rates $\frac{d \lambda}{d t}=0.0472 \mathrm{~Hz}$ to $\frac{d \lambda}{d t}=0.67 \mathrm{~Hz}$. At each stretch rate, we employ the Bayesian framework to construct the chains, marginal densities, and mean parameter values for the viscoelastic parameters given in (24), (26) and (28).

The mean viscoelastic parameters at five stretch rates ranging from $\frac{d \lambda}{d t}=$ $0.0472 \mathrm{~Hz}$ to $\frac{d \lambda}{d t}=0.67 \mathrm{~Hz}$ are respectively reported in Tables 50 for the linear Ogden model, linear nonaffine model, and nonlinear nonaffine model. We note that $\tau$ monotonically decreases for the majority of values identified regardless of which model is employed. This trend is further discussed in Section 5.3

The accuracy and predictive capabilities of the three models, with fixed hyperelastic parameters, is further illustrated by the $95 \%$ credible and prediction intervals plotted in Figures 68 see Section 4.2 for motivating discussion. The

Table 2: Mean parameter values for the linear nonaffine model estimated at the rates $\frac{d \lambda}{d t}$.

\begin{tabular}{c|c|c|c|c|c}
\hline \multicolumn{7}{c}{ Linear Nonaffine Model } \\
\hline \hline$\frac{d \lambda}{d t}$ & $G_{c}(\mathrm{kPa})$ & $\lambda_{\max }$ & $G_{e}(\mathrm{kPa})$ & $\tau(\mathrm{s})$ & $\gamma(\mathrm{kPa})$ \\
\hline \hline $6.7 \times 10^{-5} \mathrm{~Hz}$ & 8.27 & 4.66 & 17.8 & $2.38 \times 10^{5}$ & 8.86 \\
\hline $0.0472 \mathrm{~Hz}$ & 3.52 & 3.91 & 28.8 & 342.5 & 19.0 \\
\hline $0.10 \mathrm{~Hz}$ & 1.74 & 3.70 & 29.8 & 162 & 21.6 \\
\hline $0.335 \mathrm{~Hz}$ & 5.59 & 3.81 & 43.0 & 25.2 & 24.8 \\
\hline $0.50 \mathrm{~Hz}$ & 8.35 & 3.76 & 40.9 & 13.4 & 22.1 \\
\hline $0.67 \mathrm{~Hz}$ & 5.13 & 3.74 & 45.3 & 9.81 & 25.5 \\
\hline \hline
\end{tabular}


Table 3: Mean parameter values for the linear Ogden model estimated at the rates $\frac{d \lambda}{d t}$. The parameters $\mu_{i}(i=1,2,3)$ are in units $\mathrm{kPa}$ while $\alpha_{i}$ are unitless.

\begin{tabular}{c|c|c|c|c|c|c|c|c}
\hline \hline \multicolumn{7}{c}{ Linear Ogden Model } \\
\hline \hline$\frac{d \lambda}{d t}$ & $\mu_{1}$ & $\mu_{2}$ & $\mu_{3}$ & $\alpha_{1}$ & $\alpha_{2}$ & $\alpha_{3}$ & $\tau(\mathrm{s})$ & $\gamma(\mathrm{kPa})$ \\
\hline \hline $6.7 \times 10^{-5} \mathrm{~Hz}$ & 84.4 & $7.06 \times 10^{-3}$ & 1.79 & 0.045 & 2.78 & 3.11 & $4.97 \times 10^{5}$ & 16.5 \\
\hline $0.0472 \mathrm{~Hz}$ & 6.87 & $42.1 \times 10^{-3}$ & 67.8 & -3.13 & 4.86 & 0.87 & 329 & 18.4 \\
\hline $0.10 \mathrm{~Hz}$ & 0.76 & $-30.2 \times 10^{-3}$ & 63.3 & 4.68 & 0.69 & 1.03 & 91.2 & 14.0 \\
\hline $0.335 \mathrm{~Hz}$ & -65.0 & $0.21 \times 10^{-3}$ & 188 & 0.52 & 7.67 & 0.43 & 46.1 & 38.6 \\
\hline $0.50 \mathrm{~Hz}$ & -0.081 & $0.046 \times 10^{-3}$ & 145 & 0.97 & 9.36 & 0.11 & 34.0 & 42.6 \\
\hline $0.67 \mathrm{~Hz}$ & -163 & $-15.9 \times 10^{-3}$ & 258 & 1.68 & 5.21 & 1.08 & 52.9 & 93.9 \\
\hline \hline
\end{tabular}

prediction intervals, plotted in Figure 8 for the nonlinear nonaffine model, are approximately three times narrower than those for the linear Ogden and linear nonaffine models. Due to the difficulty in discerning each interval, a zoomed view of the two extreme stretch rates are plotted in Figure 9. These results further demonstrates that among the three considered models, the nonlinear nonaffine model provides the most accurate predictive capabilities as stretch

Table 4: Errors $e_{M C M C}=\frac{1}{N} \sum_{i=1}^{N}\left[s_{33}^{\text {data }}(i)-s_{33}(i ; \bar{\theta})\right]^{2}$, with units of $\mathrm{kPa}^{2}$, for the Ogden and nonaffine models. Results are delineated by those obtained when all parameters are sampled or only viscoelastic parameters are sampled with fixed hyperelastic parameters.

\begin{tabular}{c|c|c|c|c|c|c}
\hline \hline Model & Ogden & \multicolumn{2}{|c|}{ Nonaffine } & Ogden & \multicolumn{2}{c}{ Nonaffine } \\
\hline & Linear & Linear & Nonlinear & Linear & Linear & Nonlinear \\
\hline$\frac{d \lambda}{d t}$ & \multicolumn{3}{|c|}{ All } & \multicolumn{3}{c}{ Viscoelastic } \\
\hline \hline $6.7 \times 10^{-5} \mathrm{~Hz}$ & 0.50 & 0.87 & 1.86 & 0.50 & 0.87 & 1.86 \\
\hline $0.0472 \mathrm{~Hz}$ & 0.60 & 0.68 & 2.69 & 9.34 & 11.0 & 2.71 \\
\hline $0.10 \mathrm{~Hz}$ & 0.67 & 0.81 & 2.96 & 14.6 & 16.1 & 3.03 \\
\hline $0.335 \mathrm{~Hz}$ & 3.50 & 4.05 & 14.4 & 62.9 & 67.8 & 17.2 \\
\hline $0.50 \mathrm{~Hz}$ & 4.40 & 5.70 & 14.6 & 54.4 & 59.2 & 33.0 \\
\hline $0.67 \mathrm{~Hz}$ & 4.80 & 6.42 & 22.5 & 82.1 & 86.6 & 25.7 \\
\hline
\end{tabular}




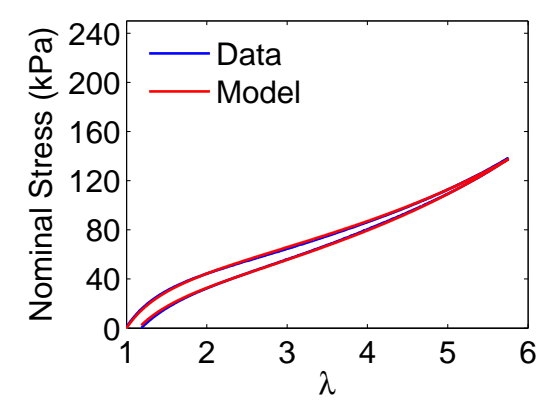

(a) $\frac{d \lambda}{d t}=6.7 \times 10^{-5} \mathrm{~Hz}$

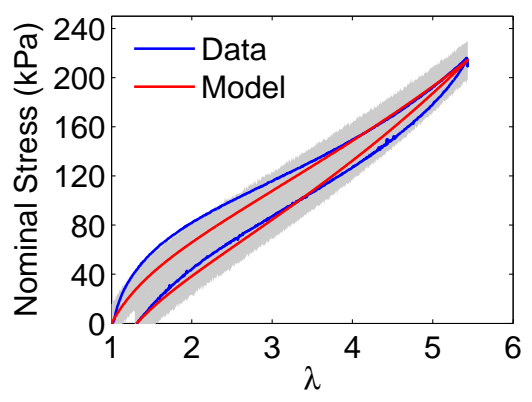

(c) $\frac{d \lambda}{d t}=0.335 \mathrm{~Hz}$

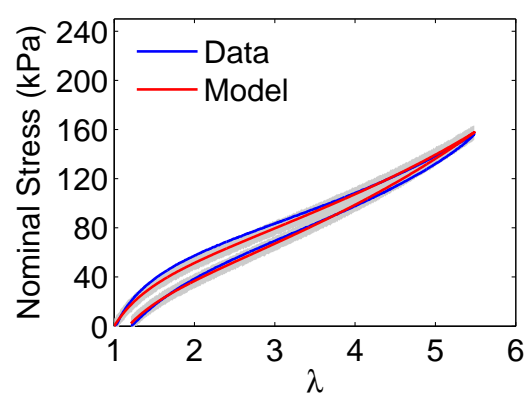

(b) $\frac{d \lambda}{d t}=0.0472 \mathrm{~Hz}$

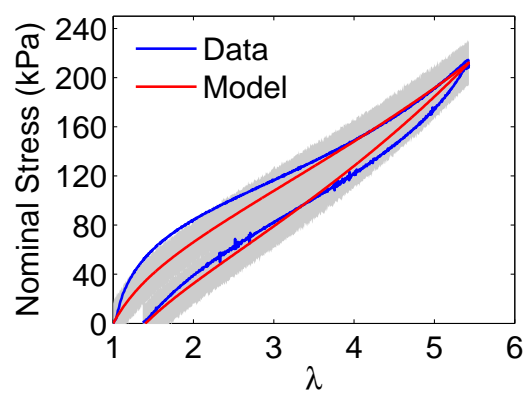

(d) $\frac{d \lambda}{d t}=0.67 \mathrm{~Hz}$

Figure 6: Credible (dark gray) and prediction (light gray) intervals for the linear Ogden model with fixed hyperelastic parameters.

rates are increased beyond the nominal values used to estimate the subsequently fixed hyperelastic parameters. For all three models, the width of the credible

Table 5: Mean viscoelastic parameters for the linear Ogden model estimated at the rates $\frac{d \lambda}{d t}$.

\begin{tabular}{c|c|c}
\hline \hline \multicolumn{3}{|c}{ Linear Ogden Model } \\
\hline \hline$\frac{d \lambda}{d t}$ & $\tau(\mathrm{s})$ & $\gamma(\mathrm{kPa})$ \\
\hline \hline $6.7 \times 10^{-5} \mathrm{~Hz}$ & $1.14 \times 10^{6}$ & 36.2 \\
\hline $0.0472 \mathrm{~Hz}$ & $1.14 \times 10^{3}$ & 43.3 \\
\hline $0.10 \mathrm{~Hz}$ & 474 & 42.9 \\
\hline $0.335 \mathrm{~Hz}$ & 108 & 58.2 \\
\hline $0.50 \mathrm{~Hz}$ & 66.9 & 61.0 \\
\hline $0.67 \mathrm{~Hz}$ & 43.2 & 58.6 \\
\hline \hline
\end{tabular}


Table 6: Mean viscoelastic parameter values for the linear nonaffine model estimated at the rates $\frac{d \lambda}{d t}$.

\begin{tabular}{c|c|c}
\hline \hline \multicolumn{3}{c}{ Linear Nonaffine Model } \\
\hline \hline$\frac{d \lambda}{d t}$ & $\tau(\mathrm{s})$ & $\gamma(\mathrm{kPa})$ \\
\hline \hline $6.7 \times 10^{-5} \mathrm{~Hz}$ & $2.38 \times 10^{5}$ & 8.84 \\
\hline $0.0472 \mathrm{~Hz}$ & 381 & 16.0 \\
\hline $0.10 \mathrm{~Hz}$ & 152 & 15.6 \\
\hline $0.335 \mathrm{~Hz}$ & 52.4 & 31.0 \\
\hline $0.50 \mathrm{~Hz}$ & 33.7 & 33.8 \\
\hline $0.67 \mathrm{~Hz}$ & 20.5 & 31.6 \\
\hline \hline
\end{tabular}

and prediction intervals increases as stretch rates are increased from the nominal values. The increased width of prediction intervals is in accordance with increased values of the inferred observation variances $\sigma^{2}$. This demonstrates the loss of predictive accuracy that is typical when extrapolating and indicates the necessity of determining validation regimes in which a model provides a specified accuracy. Finally, we note that the $95 \%$ prediction intervals contain approximately $95 \%$ of the measured data. This helps verify our use of the Gaussian likelihood (31) since the next measurement is expected to lie within this interval with $95 \%$ probability.

We noted in Section 5.1 that the nearly single-valued correlations observed

Table 7: Mean viscoelastic parameter values for the nonlinear nonaffine model estimated at the rates $\frac{d \lambda}{d t}$. Note that $\tau=\frac{\eta}{\gamma}$ which can be determined from (14) and (15).

\begin{tabular}{c|c|c|c}
\hline \hline \multicolumn{4}{c}{ Nonlinear Nonaffine Model } \\
\hline \hline$\frac{d \lambda}{d t}$ & $\tau(\mathrm{s})$ & $\gamma(\mathrm{kPa})$ & $\beta$ \\
\hline \hline $6.7 \times 10^{-5} \mathrm{~Hz}$ & $1.41 \times 10^{5}$ & 44.2 & 1.83 \\
\hline $0.0472 \mathrm{~Hz}$ & 87.3 & 55.6 & 2.76 \\
\hline $0.10 \mathrm{~Hz}$ & 37.2 & 49.3 & 2.82 \\
\hline $0.335 \mathrm{~Hz}$ & 18.3 & 65.0 & 4.29 \\
\hline $0.50 \mathrm{~Hz}$ & 32.6 & 42.4 & 4.28 \\
\hline $0.67 \mathrm{~Hz}$ & 10.3 & 54.2 & 4.39 \\
\hline \hline
\end{tabular}




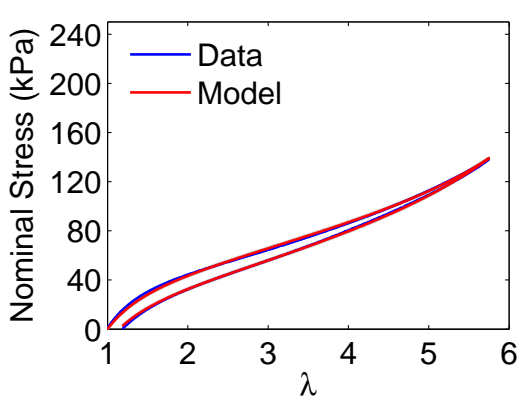

(a) $\frac{d \lambda}{d t}=6.7 \times 10^{-5} \mathrm{~Hz}$

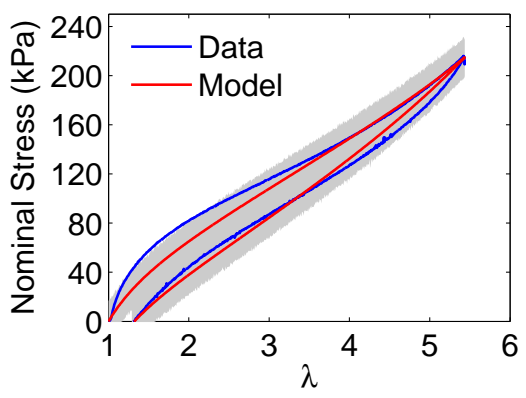

(c) $\frac{d \lambda}{d t}=0.335 \mathrm{~Hz}$

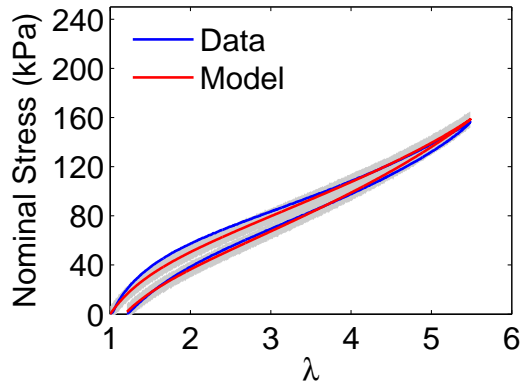

(b) $\frac{d \lambda}{d t}=0.0472 \mathrm{~Hz}$

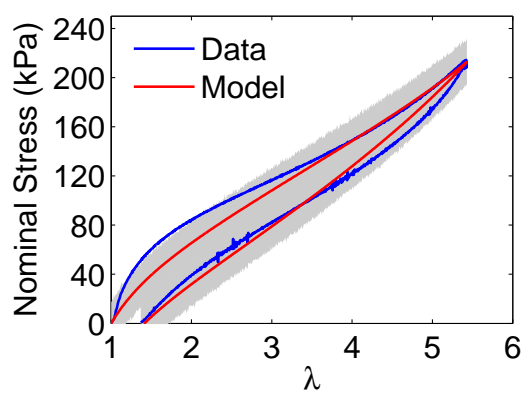

(d) $\frac{d \lambda}{d t}=0.67 \mathrm{~Hz}$

Figure 7: Credible (dark gray) and prediction (light gray) intervals for the linear nonaffine model with fixed hyperelastic parameters.

in the pairwise plots of Figure 5 indicate that the complete parameter set $\theta=$ $\left[G_{e}, G_{c}, \lambda_{\max }, \eta, \beta, \gamma\right]$ is not jointly identifiable. This provided one motivation for fixing the hyperelastic parameters at the values estimated for the slowest stretch rate.

The marginal densities and pairwise plots for the remaining viscoelastic parameters $\theta=[\eta, \beta, \gamma]$ at the fastest stretch rate $\frac{d \lambda}{d t}=0.67 \mathrm{~Hz}$ are plotted in Figures 10 and 11. The results shown for $0.67 \mathrm{~Hz}$ are representative of the other test cases. The pairwise plots illustrate that whereas the parameters are correlated, the relations are no longer nearly single-valued. Hence the parameters are identifiable and hence can be uniquely estimated from the data. Moreover, the marginal densities are approximately Gaussian. Hence the resulting model provides good predictions and has parameters that can be readily estimated. 

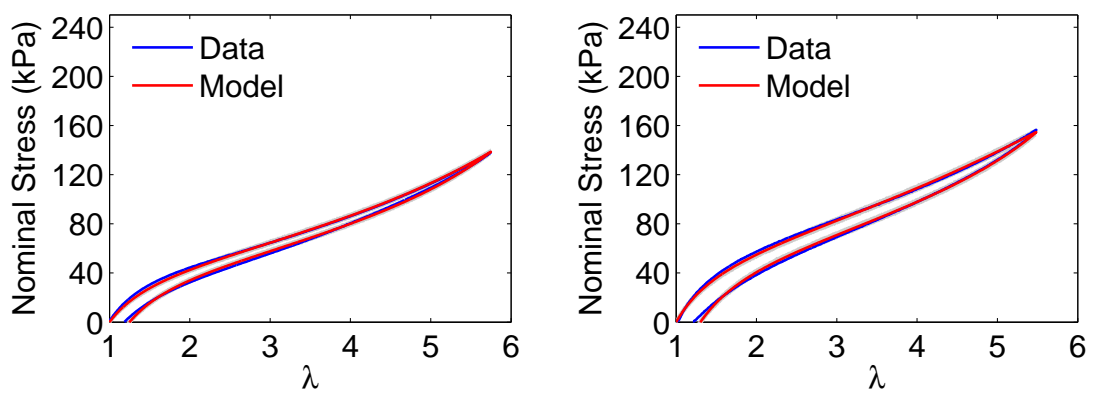

(a) $\frac{d \lambda}{d t}=6.7 \times 10^{-5} \mathrm{~Hz}$

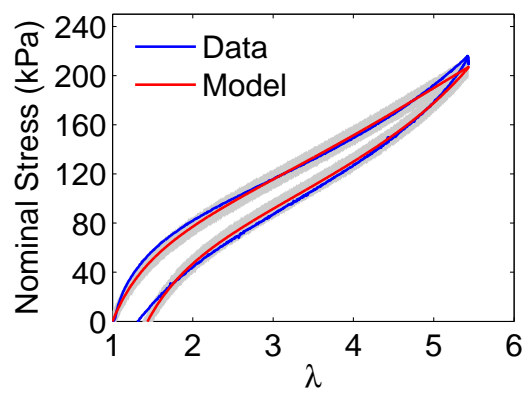

(b) $\frac{d \lambda}{d t}=0.0472 \mathrm{~Hz}$

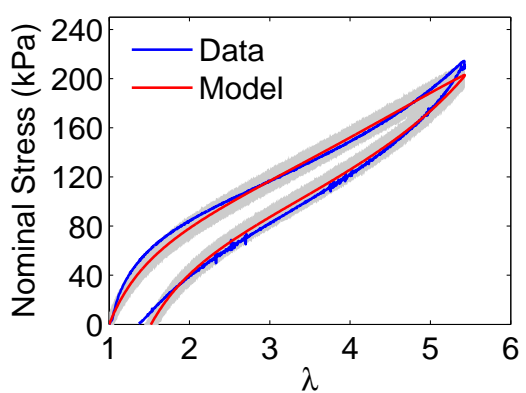

(c) $\frac{d \lambda}{d t}=0.335 \mathrm{~Hz}$

(d) $\frac{d \lambda}{d t}=0.67 \mathrm{~Hz}$

Figure 8: Credible (dark gray) and prediction (light gray) intervals for the nonlinear nonaffine model with fixed hyperelastic parameters.

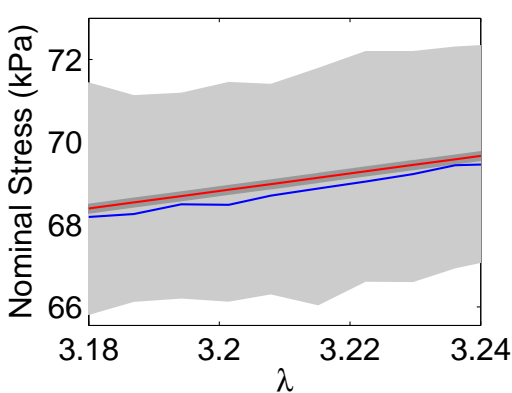

(a) $\frac{d \lambda}{d t}=6.7 \times 10^{-5} \mathrm{~Hz}$

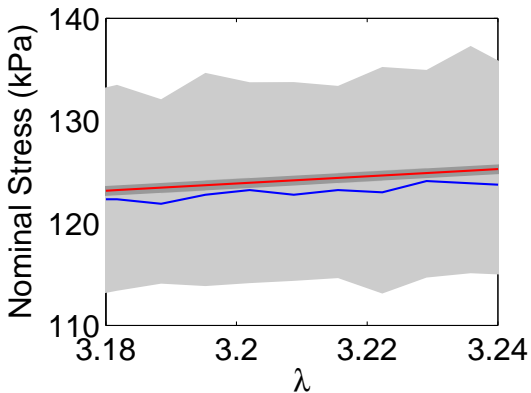

(b) $\frac{d \lambda}{d t}=0.67 \mathrm{~Hz}$

Figure 9: A zoomed in view of the credible (dark gray) and prediction (light gray) intervals from Figure 8 for the slowest and fastest stretch rate. The zoomed in view is representative of the majority of the hysteresis curve. 

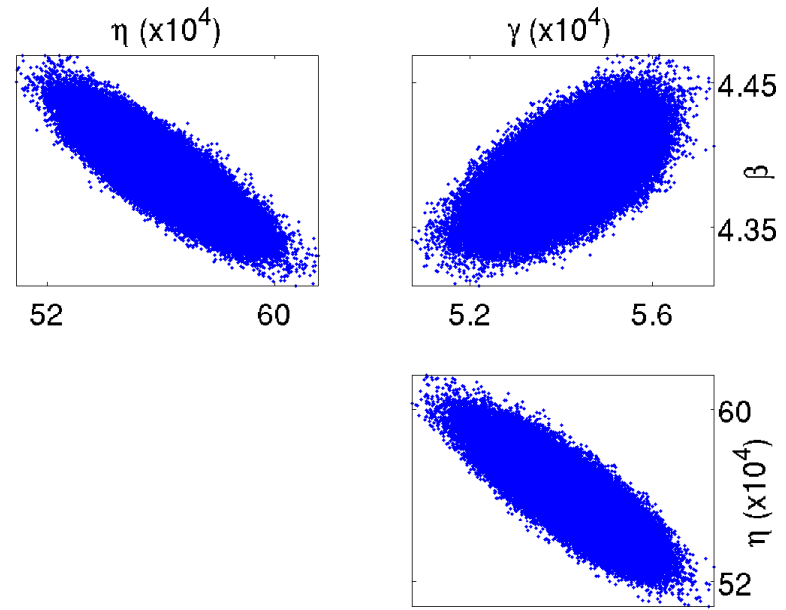

Figure 10: Joint sample points for the viscoelastic parameters in the nonlinear nonaffine model at the stretch rate $\frac{d \lambda}{d t}=0.67 \mathrm{~Hz}$.

\subsection{Power Law Fit}

It is desirable to formulate a viscoelastic model that can be applied to a broader range of stretch rates beyond the discrete set of experiments and model fits obtained here. It is known that viscoelastic polymers often exhibit power law behavior [2, Ch. 9]. By using the time constant obtained from (15) or (18), we investigate the degree to which the time constant $\tau$ obeys a power law function with respect to the stretch rate. The provides useful insight into the assumption of our Taylor expansion approximation of the entropy generation
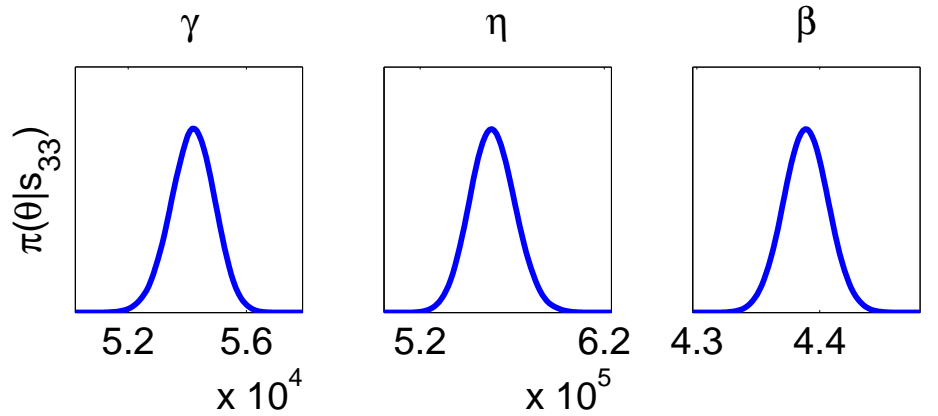

Figure 11: Marginal posterior densities for the viscoelastic parameters in the nonlinear nonaffine model at the stretch rate $\frac{d \lambda}{d t}=0.67 \mathrm{~Hz}$. 
function from (12). For simplicity, we make these estimates based on mean parameter values rather than conducting additional Bayesian analysis on the hypothesis of a power law model.

Here we investigate the degree to which the time constant $\tau$ obeys the power law

$$
\tau_{\text {model }}=A\left(\frac{d \lambda}{d t}\right)^{-B}
$$

where $A$ and $B$ are phenomenological parameters. Because we are not focused on quantifying uncertainty in these parameters, we take them to be deterministic and estimate optimal values using a Nelder-Mead algorithm.

Optimal parameter values and log-scale plots are reported in Table 8 and Figure 12. We note that $B$ exhibits little variation, 0.99-1.12, for all three models thus yielding an inverse relationship between $\tau$ and $\frac{d \lambda}{d t}$. As expected from the prediction intervals, Figure 12 illustrates that the largest variation occurs for large stretch rates $\frac{d \lambda}{d t}$.

\section{Concluding Remarks}

Viscoelastic models have been applied to characterize rate-dependent deformation of elastomers that undergo large deformation. Whereas Bayesian statistics have been applied in a number of fields such as weather forecasting, fluid dynamics, and atomistic modeling, limited uncertainty analysis has been

Table 8: Parameters for the power law (32) with all parameters sampled and only viscoelastic parameters sampled.

\begin{tabular}{c|c|c|c|c}
\hline \hline Sampling & \multicolumn{2}{|c|}{ All } & \multicolumn{2}{c}{ Viscoelastic } \\
\hline & A & B & A & B \\
\hline \hline Linear Ogden & 10.1 & 1.12 & 36.1 & 1.08 \\
\hline Linear Nonaffine & 15.9 & 1.00 & 18.1 & 0.99 \\
\hline Nonlinear Nonaffine & 3.04 & 1.12 & 2.94 & 1.12 \\
\hline \hline
\end{tabular}




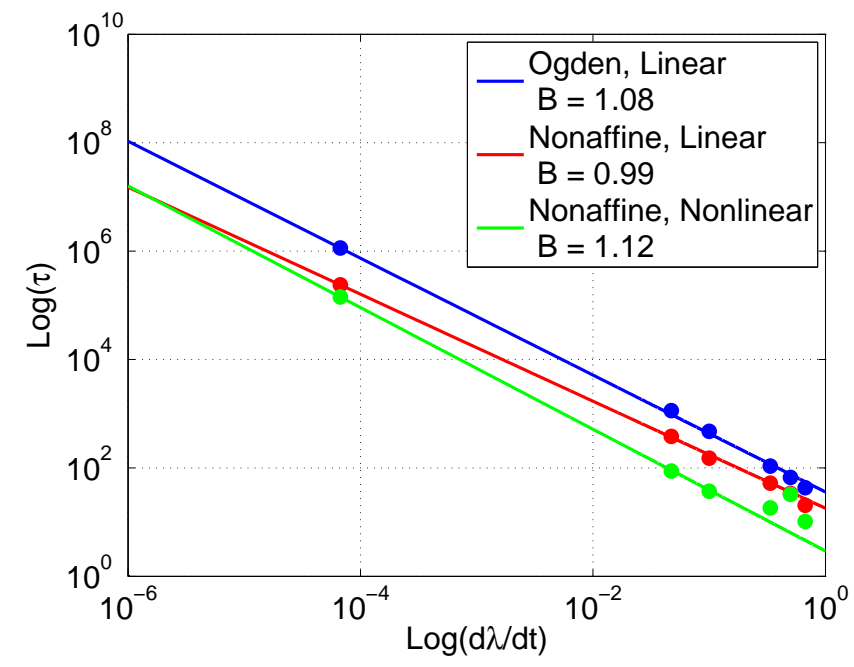

Figure 12: Log-scale plot of the viscoelastic time constants using power law fit (32).

applied to developing solid mechanics constitutive models. Key results in this analysis have shown how Bayesian statistics can be used to quantify the uncertainty of viscoelastic constitutive relations and error propagation when it is integrated into a model to predict stress over a range of stretch rates. In comparing two hyperelastic energy functions, we show similar results between a six parameter Odgen model and a three parameter nonaffine model, justifying the use of the lower order nonaffine model based on uni-axial stress-stretch data.

The experimental analysis of VHB 4910 has illustrated significant ratedependent deformation thus motivating the need to couple hyperelasticity with viscoelastic dissipative functions. We have focused on a viscoelastic model that assumes the complex polymer network viscous losses can be modeled by dissipative functions that are proportional to the neo-Hookean model (linear viscoelasticity) or proportional to the nonaffine hyperelastic function (nonlinear viscoelasticity). Both linear and nonlinear viscoelastic models were calibrated 565 using experiments. It was shown that the nonlinear viscoelastic model quantified the rate-dependent deformation of VHB with the greatest accuracy, as it accounted for the behavior at all stretch rates with a single set of hyperelastic 
parameters. The errors from calibrating both hyperelastic and viscoelastic parameters gave the lowest error; however, these results were deemed non-physical since the hyperelastic parameters must be independent of stretch rate. In addition, strong correlation among all the nonaffine material parameters (excluding $\left.\lambda_{\max }\right)$ were shown in Figure 5. This suggests further model reduction may be possible by identifying how polymer entanglement and crosslinked density gives rise to dissipation. Testing under multi-axial loading and on other elastomers should be conducted to determine if there are general trends in parameter correlation among other elastomer compositions. Lastly, by fixing the hyperelastic parameters and identifying the viscoelastic time constants over a range of stretch rates, estimates on the predictive capabilities over stretch rates from $6.7 \times 10^{-5} \mathrm{~Hz}$ to $0.67 \mathrm{~Hz}$ were obtained. A power exponent close to one was found to reasonably support the data over this range of stretch rates.

\section{Acknowledgements}

The research of RCS has been supported in part by the Air Force Office of Scientific Research (AFOSR) through the grant AFOSR FA9550-11-1-0152 and the National Science Foundation (NSF) through the Collaborative Research grant CMMI-1306290. WSO, MRH, and PRM gratefully acknowledge support from an Army Research Laboratory Collaborative Technology Alliance (R-CTA) and the Army Research Office through grant W911NF-13-1-0146, program manager Bryan Glaz. WSO also appreciates NSF support through the Collaborative Research CMMI grant 1306320. The authors gratefully appreciate the excellent comments provided by one of the reviewers.

\section{Bibliography}

[1] R. Smith, Smart Material Systems: Model Development, SIAM, Philadelphia, PA, 2005.

[2] M. Rubinstein, R. Colby, Polymer Physics, Oxford University Press, Oxford, 2003. 
[3] A. Drozdov, Mechanics of Viscoelastic Solids, John Wiley \& Sons, Inc., London, 1998.

[4] J. Bergström, M. Boyce, Constitutive modeling of the large strain timedependent behavior of elastomers, J. Mech. Phys. Solids 46 (5) (1998) 931-954.

[5] R. Christensen, A nonlinear theory of viscoelasticity for application to elastomers, Trans. ASME AMD Winter Annual Meeting 47 (1980) 762-768.

[6] S. Edwards, Dynamics of entangle polymers, Proc. R. Soc. Lond. A 385 (1982) 267-288.

[7] G. Holzapfel, On large strain viscoelasticity: Continuum formulation and finite element applications to elastomeric structures, Int. J. Num. Meth. Eng. 39 (1996) 3903-3926.

[8] J. Simo, On a fully three-dimensional finite-strain viscoelastic damage model: Formulation and computational aspects, Comput. Meth. Appl. Mech. Engrg. 60 (1987) 153-173.

[9] H. Hilton, S. Yi, The significance of (an)isotropic viscoelastic Poisson ratio stress and time dependencies, Int. J. Solids Struct. 35 (23) (1998) 30813095 .

[10] G. A. Holzapfel, J. C. Simo, A new viscoelastic constitutive model for continuous media at finite thermomechanical changes, Int. J. Solids Struct. 33 (20-22) (1996) 3019-3034. doi:http://dx.doi.org/10.1016/0020-7683(95) 00263-4.

[11] M. Gurtin, Thermodynamics with internal state variables, J. Chem. Phys. 47 (1967) 597-613.

${ }_{620}[12]$ S. Peng, K. Valanis, R. Landel, Nonlinear viscoelasticity and relaxation phenomena of polymer solids, Acta Mechanica 25 (1977) 229-240. 
[13] G. Holzapfel, T. Gasser, A viscoelastic model for fiber-reinforced composites at finite strains: Continuum basis, computational aspects and applications, Comput. Methods Appl. Mech. Engrg. 190 (2001) 4379-4403.

[14] L. Treloar, The Physics of Rubber Elasticity, Third Edition, Oxford University Press, Oxford, 1975.

[15] G. Holzapfel, Nonlinear Solid Mechanics, John Wiley \& Sons, Inc., Chichester, 2000.

[16] J. Davidson, N. Goulbourne, A nonaffine network model for elastomers undergoing finite deformations, J. Mech. Phys. Solids 61 (8) (2006) 17841797.

[17] M. Rubinstein, S. Panyukov, Elasticity of polymer networks, Macromol. 35 (2002) 6670-6686.

[18] H. Haario, M. Laine, A. Mira, E. Saksman, DRAM: Efficient adaptive MCMC, Statistics and Computing 16 (4) (2006) 339-354.

[19] R. Smith, Uncertainty Quantification: Theory, Implementation, and Applications, SIAM, Philadelphia, PA, 2014.

[20] S. L. Frederiksen, K. W. Jacobsen, K. S. Brown, J. P. Sethna, Bayesian ensemble approach to error estimation of interatomic potentials, Phys. Rev. Lett. 93 (2004) 165501.

[21] A. Croicu, M. Y. Hussaini, A. Jameson, G. Klopfer, Robust airfoil optimization using maximum expected value and expected maximum value approaches, AIAA J. 50 (9) (2012) 1905-1919.

[22] D. Wilks, Statistical Methods in the Atmospheric Sciences: An Introduction, Academic Press, San Diego, 1995.

[23] A. Bernardini, F. Tonon, Bounding Uncertainty in Civil Engineering, Springer-Verlay Berline Heidelberg, 2010. 
[24] S. Mahadevan, A. Haldar, Probability, reliability and statistical method in engineering design, John Wiley \& Sons, 2000.

[25] H. Zhengzheng, R. Smith, N. Burch, M. Hays, W. Oates, A modeling and uncertainty quantification framework for a flexible structure with macrofiber composite actuators operating in hysteretic regimes, J. Intell. Mater. Sys. Struct. 25 (2) (2014) 204-228. doi:10.1177/1045389X13489781.

${ }_{655}$ [26] W. Oates, A quantum informed continuum model for ferroelectric materials, Smart Materials and Structures 23 (10) (2014) 104009.

URL http://stacks .iop.org/0964-1726/23/i=10/a=104009

[27] H. Haario, E. Saksman, J. Tamminen, An adaptive metropolis algorithm, Bernoulli 7 (2) (2001) 223-242.

660 [28] M. Laine, Dram website URL http://helios.fmi.fi/ lainema/dram/

[29] M. Golberg, H. Cho, Introduction to Regression Analysis, WITPress, Southhampton, 2004.

[30] A. Gelman, J. Carlin, H. Stern, D. Rubin, Bayesian Data Analysis, 2nd Edition, Chapman and Hall/CRC, Boca Raton, FL, 2004. 